Short Communication

\title{
Inv21p12q22del21q22 and intellectual disability
}

\author{
Renata Oliveira a,*,1, Sofia Dória ${ }^{a}$, Carmen Madureira ${ }^{a}$, Vera Lima a , Carolina Almeida a , Maria J. Pinho a , \\ Carla Ramalho $^{\text {b }}$, Eunice Matoso ${ }^{\mathrm{c}, \mathrm{d}}$, Alberto Barros ${ }^{\mathrm{a}}$, Isabel M. Carreira ${ }^{\mathrm{c}, \mathrm{d}}$, Carla P. Moura ${ }^{\mathrm{a}}$ \\ a Dept. of Human Genetics, Faculty of Medicine, University of Porto/Hospital São João, Portugal \\ ${ }^{\mathrm{b}}$ Dept. of Obstetrics/Gynecology, Hospital São João, Porto, Portugal \\ c Laboratory of Cytogenetics and Genomics, Faculty of Medicine, University of Coimbra, Portugal \\ d Laboratory of Citogenetics, Pediatric Hospital Carmona da Mota, Coimbra, Portugal
}

\section{A R T I C L E I N F O}

\section{Article history:}

Accepted 2 December 2012

Available online $\mathrm{xxxx}$

\section{Keywords:}

Chromosome 21

Pericentric inversion

Intellectual disability

ArrayCGH

\begin{abstract}
A B S T R A C T
Chromosomal rearrangements are common in humans. Pericentric inversions are among the most frequent aberrations (1-2\%). Most inversions are balanced and do not cause problems in carriers unless one of the breakpoints disrupts important functional genes, has near submicroscopic copy number variants or hosts "cryptic" complex chromosomal rearrangements. Pericentric inversions can lead to imbalance in offspring. Less than 3\% of Down syndrome patients have duplication as a result of parental pericentric inversion of chromosome 21 . We report a family with an apparently balanced pericentric inversion of chromosome 21 . The proband, a 23-year-old female was referred for prenatal diagnosis at 16 weeks gestation because of increased nuchal translucency. She has a familial history of Down's syndrome and moderate intellectual disability, a personal history of four spontaneous abortions and learning difficulties. Peripheral blood and amniotic fluid samples were collected to perform proband's and fetus' cytogenetic analyses. Additionally, another six family members were evaluated and cytogenetic analysis was performed. Complementary FISH and MLPA studies were carried out. An apparent balanced chromosome 21 pericentric inversion was observed in four family members, two revealed a recombinant chromosome 21 with partial trisomy, and one a full trisomy 21 with an inverted chromosome 21. Array CGH analysis was performed in the mother and the brother's proband. MLPA and aCGH studies identified a deletion of about $1.7 \mathrm{Mb}$ on the long arm of inverted chromosome 21q22.11. We believe the cause of the intellectual disability/learning difficulties observed in the members with the inversion is related to this deletion. The recombinant chromosome 21 has a partial trisomy including the DSCR with no deletion. The risk for carriers of having a child with multiple malformations/intellectual disability is about 30\% depending on whether and how this rearrangement interferes with meiosis.
\end{abstract}

(C) 2012 Elsevier B.V. All rights reserved.

\section{Introduction}

Chromosomal rearrangements are common in humans and pericentric inversions (PI) are among the most frequent aberrations (frequency of about 1-2\%; Kaiser, 1984). The relation between intellectual disability (ID) and apparently balanced chromosomal rearrangements has been postulated for more than forty years (Funderburk et al., 1977). Most inversions are balanced and do not cause phenotype in carriers unless: one or both of the breakpoints disrupts an important functional gene; one breakpoint includes or is near submicroscopic copy number variants (CNVs); it hosts

Abbreviations: Array CGH, array comparative genomic hybridization; CNVs, copy number variants; DS, Down syndrome; FISH, fluorescence in situ hybridization; ID, intellectual disability; MLPA, multiplex-ligation probe amplification; OMIM, Online Mendelian Inheritance in Man; PI, pericentric inversion.

* Corresponding author. Tel.: +351 239480 638, fax: + 351239717216 .

E-mail address: renata.ro@chc.min-saude.pt (R. Oliveira).

1 The author's permanent address: Dept. of Medical Genetics, Pediatric Hospital Carmona da Mota. Postal address: Av. Afonso Romão, 3000-602 Coimbra, Portugal. "cryptic" complex chromosomal rearrangements; or there is an unidentified genetic or environmental factor (Gijsbers et al., 2010). It has been demonstrated that subtle chromosomal abnormalities are common in individuals with ID. Also, unbalanced derivatives segregate with cognitive impairment and, if there is more than one affected offspring with concordant phenotypes, it probably indicates the same unbalanced rearrangement (Knight et al., 1999).

Like other chromosomal rearrangements, PI can lead to imbalance in offspring. During meiosis of a pericentric inverted chromosome there is a risk for recombinants with partial duplication accompanied by partial deletion-a duplication of the region outside the inverted segment of one arm with a deletion of the terminal segment on the other arm. Therefore in a zygote with a partial trisomy or monosomy recombinant chromosomes, distal to the breakpoints, can occur. Down syndrome (DS) patients with a trisomy of the distal segment of chromosome 21 as a result of a parental PI of chromosome 21 are rare (Ruhi et al., 2001). As far as we know, only 14 cases of DS with recombinant chromosome 21 from parental pericentric inversion (mostly maternal) were reported so far (Fraisse et al., 1986; Gray et 
।

III

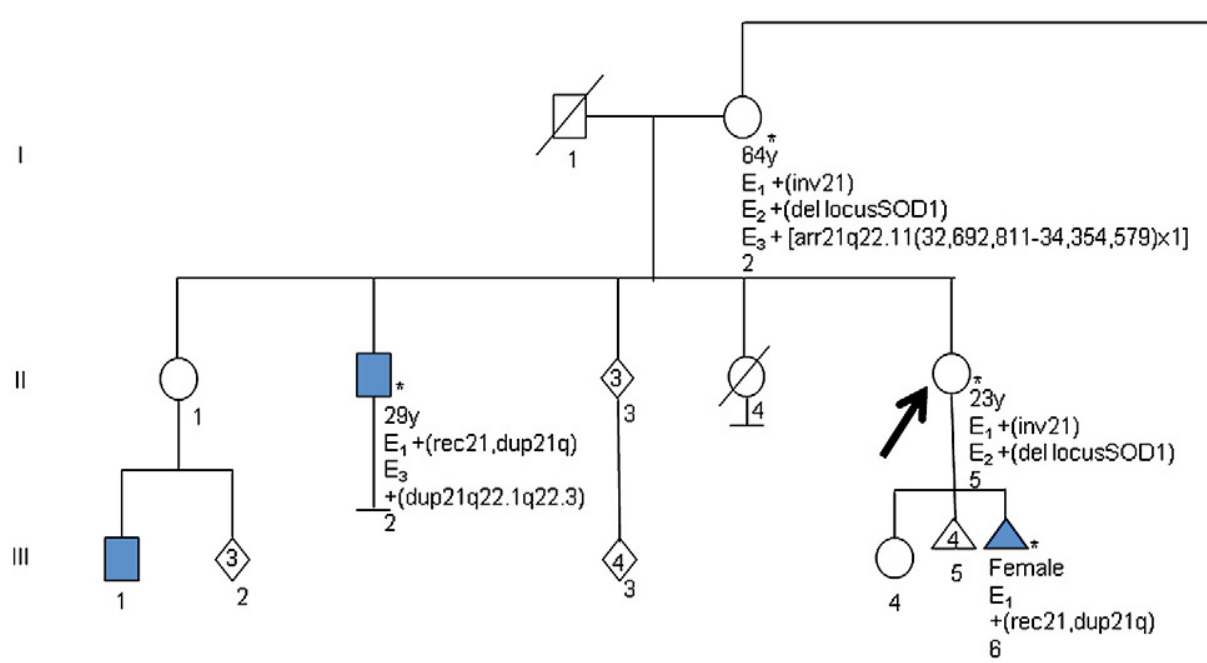

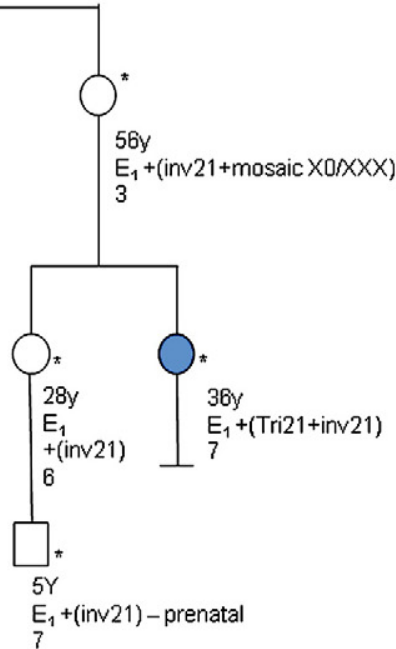

Down Syndrome (with and without karyotype testing)

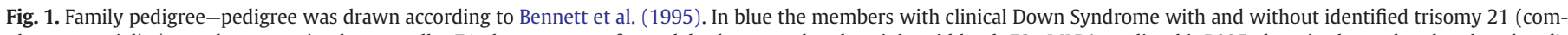

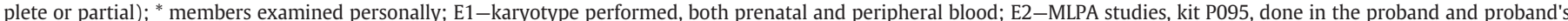

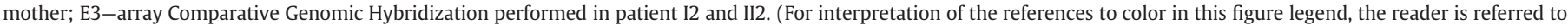
the web version of this article.)

- $\mathrm{I} 2-46, \mathrm{XX}, \operatorname{inv}(21)(\mathrm{p} 12 \mathrm{q} 22.1)$

- I3-45,X,inv(21)(p12q22.1)[6]/47,XXX,inv(21)(p12q22.1)[1]/46,XX,inv(21)(p12q22.1)[51]

- II2-46,XY,rec(21)dup(21q)inv(21)(p12q22.1)mat

- II5 (proband)-46,XX,inv(21)(p12q22.1)mat

- II6-46,XX,inv(21)(p12q22.1)mat

- II7-47,XX, +21,inv(21)(p12q22.1)mat

- III6 (proband fetus)-46,XX,rec(21)dup(21q)inv(21)(p12q22.1)mat

- III7-46,XY,inv(21)(p12q22.1)mat.

al., 1962; Lazzaro et al., 2001; Leonard et al., 1986; Miyazaki et al., 1987; Ruhi et al., 2001; Tardy et al., 1997).

In this paper, a family with moderate to severe intellectual disability, with some presenting different chromosome imbalances is described. There are members with an apparent balanced pericentric inversion involving chromosome 21 with an extra submicroscopic interstitial deletion (1.7 Mb), one member with DS with a recombinant chromosome 21 (with distal duplication of $21 \mathrm{q}$ without the interstitial deletion) and another DS patient with full trisomy 21 of which one chromosome is the inverted one.

\section{Clinical report}

The proband, a 23-year-old Caucasian female, was referred for amniotic fluid sampling at 16 weeks gestation because of increased nuchal translucency (at 12 weeks). She has a personal history of learning difficulties and four spontaneous miscarriages and a familial history of DS and moderate ID.

Additionally to the proband and the fetus, six family members were studied (pedigree, Fig. 1): the mother, the brother (with clinical DS) and a maternal aunt with her two daughters (one of which was also with clinical DS $)^{2}$ and granddaughter.

The proband has been a healthy individual. She and her mother share very similar dysmorphic features (Fig. 2) like brachychephaly with normal head circumference (occipitofrontal circumference), low anterior hairline with widow's peak, flat face, slight bifrontal

\footnotetext{
2 The aunt and the cousins had been previously studied (four years before) and then connected to this family. One of the cousins was investigated, at the time with a pregnancy in course, after her sisters' karyotype has revealed a full trisomy 21 with PI in one chromosome 21.
}

narrowing, bilateral epicanthal fold, depressed nasal bridge with bulbous nose, apparent long ears with prominent superior crus of antihelix and large lobe, and short neck. The proband also presents thick and everted vermillion of the upper lip. Their hands are broad with apparent brachydactyly. They both have moderate intellectual disability ${ }^{3}$ but functional for daily life activities. A severe social problem is present and this family live from social support.

A karyotype of peripheral blood lymphocytes and from amniotic fluid cells was performed on GTL-banded preparations in all mentioned family members. FISH studies were carried out according to standard methods, using LSI and subtelomeric 21 probes (D21S259, D21S341, D21S342 and TelVysion 21q, Abbott $\left.{ }^{\circledR}\right)$.

The cytogenetic analysis in the fetus' proband revealed the presence of a 46,XX,rec(21)dup(21q)inv(21)(p12q22.1) karyotype, and a proband 46,XX,inv(21)(p12q22.1) karyotype. Her mother's and brother's karyotypes are, respectively: 46,XX,inv(21)(p12q22.1) and 46,XY,rec(21)dup(21q)inv(21)(p12q22.1)mat.

Considering both, karyotype and FISH results, the proband has a maternal inherited apparently balanced PI of chromosome 21. Her fetus presents the derivative chromosome, an unbalanced recombinant chromosome 21, resulting in a partial trisomy 21 . The karyotype with this recombinant chromosome is similar to the one present in her brother's karyotype. The proband decided to continue the pregnancy in question that terminated in fetal death at 20 weeks of gestation.

Furthermore, we have a familial apparently balanced PI, as we could relate this same inverted chromosome to and reach other family members previously studied in our department.

\footnotetext{
${ }^{3}$ There was not possible to perform a formal cognitive evaluation neither to the proband nor to her mother, because they abandon follow-up.
} 


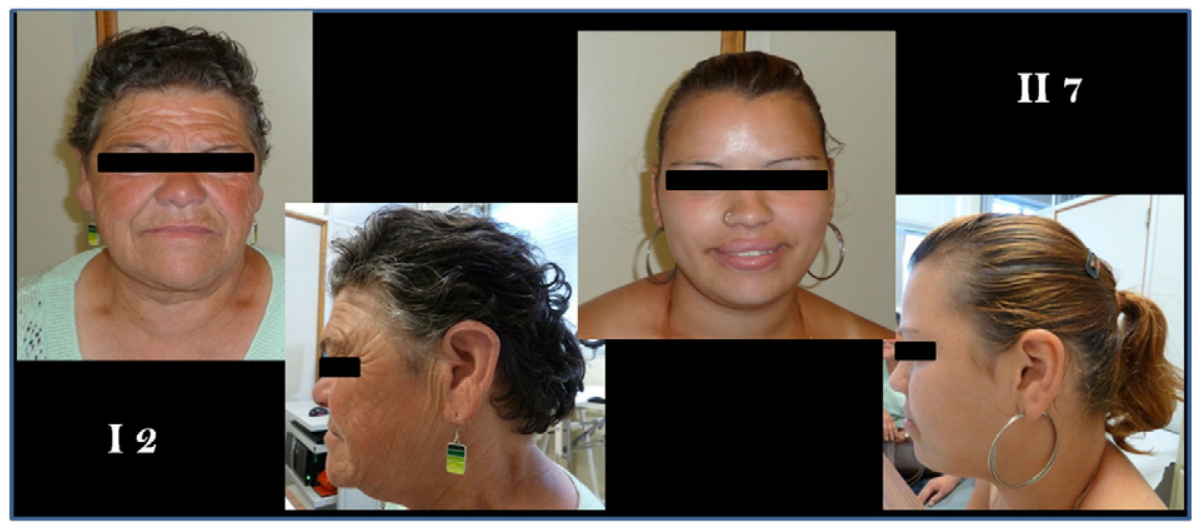

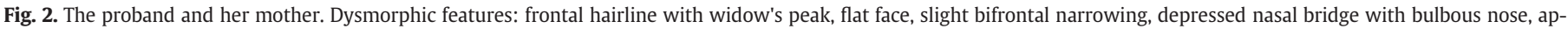

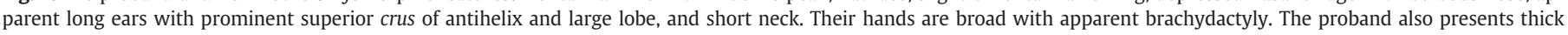
and everted vermillion of the upper lip.

In accordance to pedigree (Fig. 1), the karyotypes of the other family members found are shown

- I3-45,X,inv(21)(p12q22.1)[6]/47,XXX,inv(21)(p12q22.1)[1]/46, $\mathrm{XX}, \operatorname{inv}(21)(\mathrm{p} 12 \mathrm{q} 22.1)[51]$

- II6-46,XX,inv(21)(p12q22.1)mat

- II7-47,XX, +21,inv(21)(p12q22.1)mat

- III7-46,XY,inv(21)(p12q22.1)mat

Through conventional karyotype and FISH studies we were unable to define whether the inverted chromosome 21 was balanced or unbalanced. In order to detect possible cryptic imbalances in the breakpoints of this PI that could justify the ID observed in the proband and her mother (as well as in the cousin and aunt), a Multiplex-Ligation Probe Amplification (MLPA) study was performed in the proband, her mother and brother and an array-Comparative Genomic Hybridization (aCGH) analysis, using Agilent ${ }^{\circledR}$ 60mer oligonucleotide-array $180 \mathrm{k}$, was performed in the DNA of the proband's mother and brother.

Informed consent was obtained from the individuals involved.

\section{Results}

The MLPA analysis revealed a deletion between 21q11.2 and 21q22.3, concerning SOD1 locus, in the proband and her mother (image not shown). The same deletion was not present in the brother with DS.

A deletion of about $1.7 \mathrm{Mb}$ on the long arm of chromosome $21 \mathrm{q} 22.11$ was found on the arrayCGH [arr 21q22.11(23,692,811$34,454,579) \times 1$ ] of the proband's mother (Fig. 3). It includes 19 genes, two of which were reported in the OMIM Genetic Morbid Map (OMIM ID: SOD1, 147450; MRAP, 609196).

The proband's brother, which was shown to have a recombinant chromosome 21, is a carrier of a partial trisomy of chromosome 21q22.1q22.3, involving the Down Syndrome Critical Region (DSCR), without the deletion observed in the mother [arr 21q22.1q22.3(34,466,182-48,090,317) ×3] (Fig. 4).

From the family history we can assume that the inverted chromosome 21 co-segregates with the deletion, so it is in the same derivative.

\section{Discussion and conclusion}

High-resolution array platforms allow for detection of CNVs in carriers of apparently balanced chromosome abnormalities. The apparently balanced PI of chromosome 21 present in this family was found to have a submicroscopic rearrangement [1.7 Mb deletion] near one of the breakpoints of the inversion. This deletion might contribute to the moderate ID observed in the proband, her mother and in the other carrier family members, as well as for the phenotypic facial characteristics they share. A formal cognitive assessment could have been of great interest to better characterize the mental impairment observed in these patients. Unfortunately, it was not possible as the proband and her mother abandoned follow-up. Given their consistent refusal plus the social situation and limited ability to comprehend the implication of these results, it has been difficult to reestablish contact.

The presence of mental illness has long been related with some chromosome 21 abnormalities, and history of psychiatric disorders like bipolar disorder, with learning disability were associated to deletions of the short arm of chromosome 21 (El-Badramany et al., 1989).

The phenotypic and molecular analysis of patients with partial monosomy 21 has been reported before (Chettouh et al., 1995; Roberson et al., 2011; Valero et al., 1999), but none, as far as we know, were associated to a chromosome pericentric inversion. The majority of these partial monosomies is de novo and reported on cytogenetic level, and only a few are submicroscopic deletions.

Partial chromosome 21 monosomy is rare in human disease with variable clinical appearances due to different genomic content

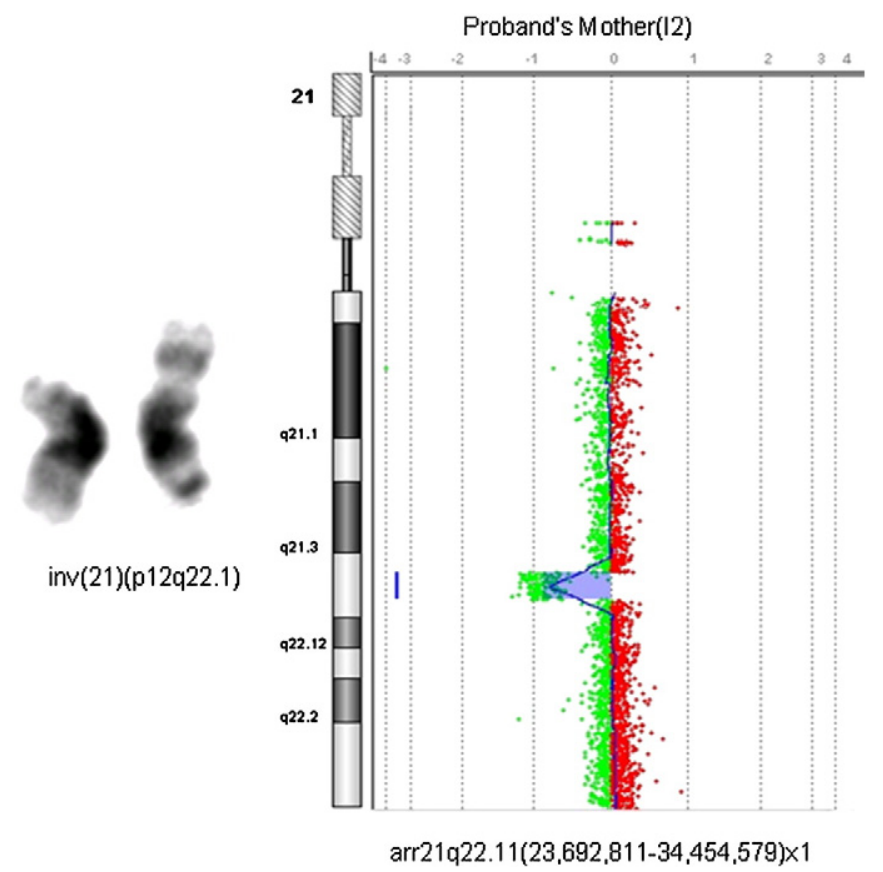

Fig. 3. Inverted chromosome $21[\operatorname{inv}(21)(\mathrm{p} 12 \mathrm{q} 22.1)$ on the right, plus arrayCGH result of I2 (the mother) showing $1.7 \mathrm{Mb}$ deletion [arr 21q22.11(23,692,811-34,454,579)×1] 


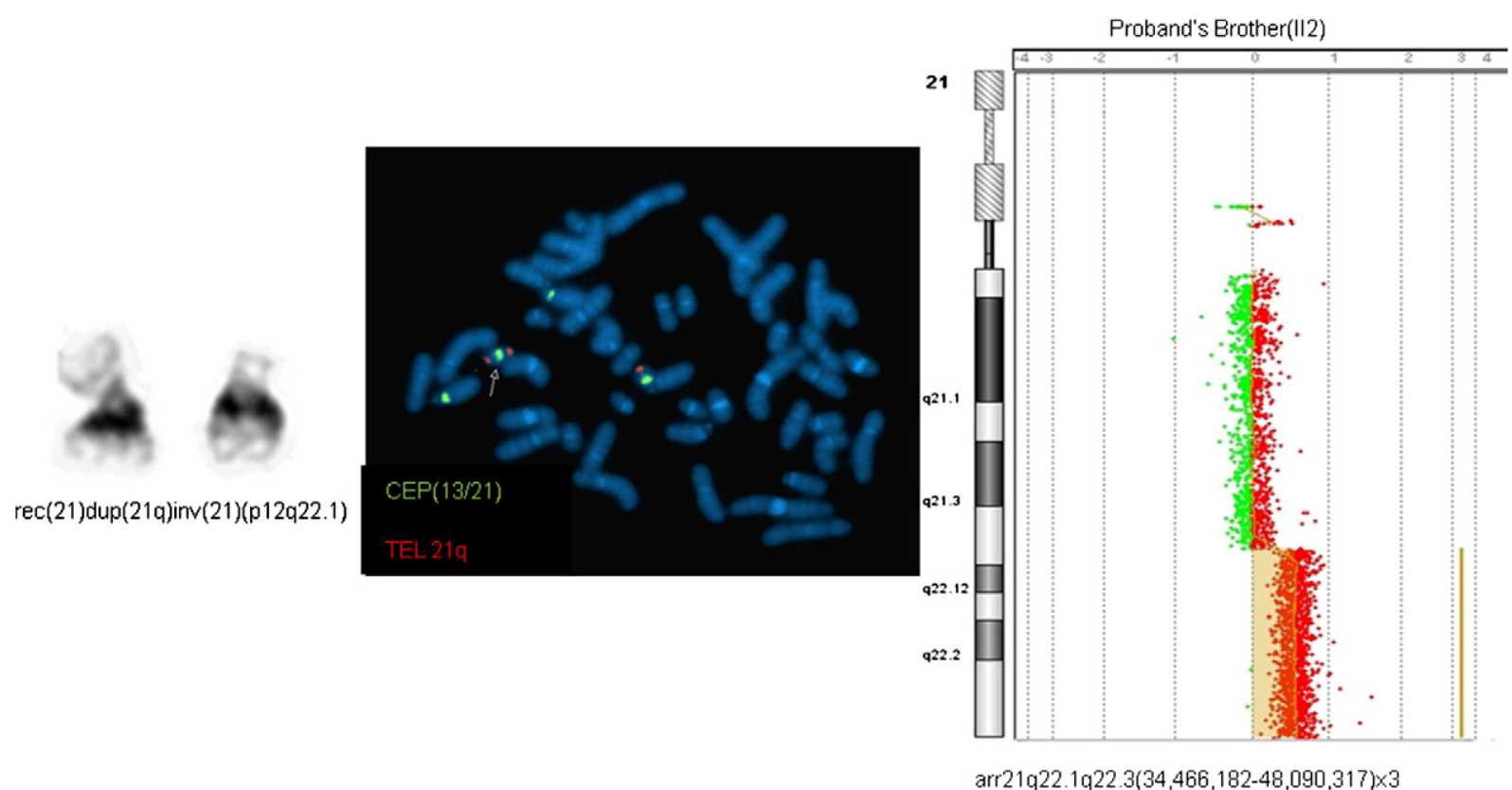

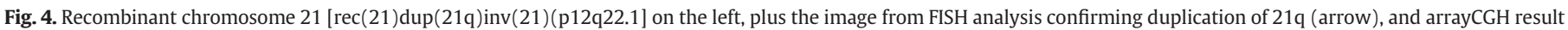
revealing duplication of II2 [arr 21q22.1q22.3 $(34,466,182-48,090,317) \times 3$ ]

resulting in phenotypes including intellectual disability, brain dysgenesis, extensive dysmorphic features (microcephaly, short neck, epicanthic folds, flat nasal bridge, low set/malformed ears, highly arched palate, and transverse palmar crease), heart and/or renal defects (Migdalska et al., 2012; Roberson et al., 2011).

The deleted region of chromosome 21(q22.11) here reported, has been referred as mapping genetically between APP-SOD1 genes, being a critical region responsible for most 21 monosomy associated traits (Valero et al., 1999). Also, other genes involved (SYNJ1, OLIG 1/2) code for glutamate receptors, interferes in synaptic transmission and in oligodendrocyte function, and together are responsible in the central nervous system for the formation and functioning of a large number of neuronal circuits. These might also contribute to the pathogenesis of intellectual disability when in haploinsufficiency (Chettouh et al., 1995). As in other similar abnormalities, differences in the genomic content of the deletion and the region spanning from APP to SOD1 would account for the phenotypic variability observed (Valero et al., 1999). The deleted region 21(q22.11) found in the family reported here includes two genes which are part of the OMIM Genetic Morbid Map (OMIM ID: SOD1, 147450; MRAP, 609196). In haploinsufficiency those genes probably are not responsible for the phenotype alone; instead it could be explained by the global interaction of other genes involved in neurotransmission, splicing and transcription of mRNA. To understand the genotype-phenotype correlation would be important to elucidate the consequence of this and other particular heterozygous deletions.

From the three family members with Down syndrome, two of them were studied: one has a regular pattern of trisomy 21 (including the inverted deleted chromosome) and the other (the proband's brother) has a recombinant chromosome 21 with a partial trisomy of DSCR without the interstitial deletion. The same recombinant form was found in the prenatal karyotype, whose gestation spontaneously terminated. Pairing in meiosis constitutes the main problem in
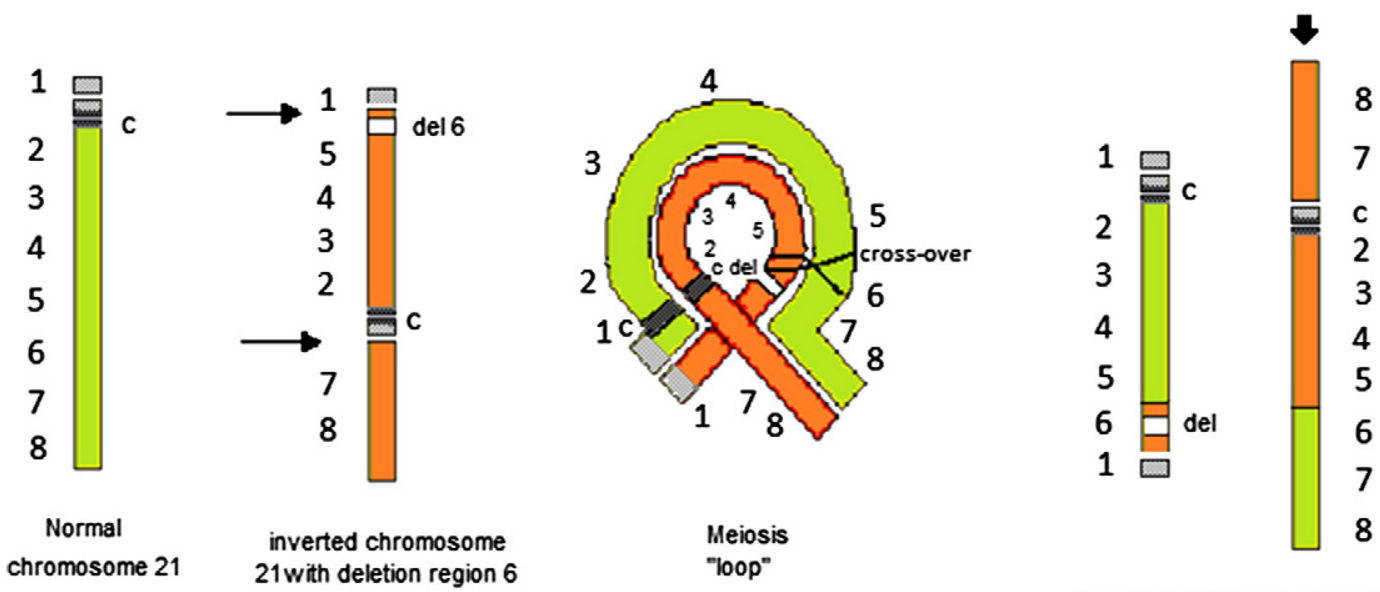

Recombinant chromosomes

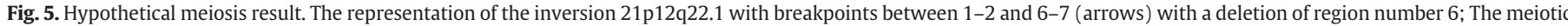

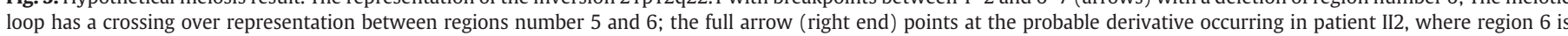
present, not including the deletion observed in the mother (identified by MLPA and, more precisely defined by aCGH studies). 
inversion heterozygosis, because crossover events (during the pachytene stage of meiosis I) within the inversion segment can lead to two monocentric recombinants with reciprocal duplications/deficiencies in the gametes, ending up in a risk of inheriting such an imbalance when conception occurs. The recombinant possibilities from a crossing over within the loop during meiosis are shown in Fig. 5, where a likely condition occurring in our recombinant patient (the brother-II2) is pointed out, overcoming the deletion observed in his mother and sister (the proband), as identified by MLPA and more precisely, by aCGH studies. Thus, the deleted region in the mother (pedigree I2), represented in our meiosis scheme by number 6 , is balanced in the brother (pedigree II2).

In conclusion, variability in biological mechanisms and phenotypes is a big challenge for genetic counseling and for laboratory pre-natal diagnosis as the risk for carriers of a pericentric inversion for miscarriages, infertility and/or chromosomally unbalanced offspring is high. As for the present familial pericentric inversion, the risk of having a child with multiple malformations/intellectual disability is about 30\% (risk estimated via hc-forum.net, Human Genetic Forum ${ }^{\circledR}$ ) depending on whether and how this rearrangement interferes with meiosis. To our knowledge, our family is the first reported to have a submicroscopic interstitial deletion of chromosome 21 associated to a pericentric inversion of it, with a probable causative phenotype of moderate intellectual disability and dysmorphic features.

\section{Disclosure statement}

None of the authors has any conflict of interest to disclose. There was no financial funding for this report.

\section{References}

Kaiser, P., 1984. Pericentric inversions. Problems and significance for clinical genetics. Hum. Genet. 68 (1), 1-47.

Funderburk, et al., 1977. Mental retardation associated with "balanced" chromosome rearrangements. Am. J. Hum. Genet. 29, 136-141.

Gijsbers, A.C.J., et al., 2010. Additional cryptic CNVs in mentally retarded patients with apparently balanced karyotypes. Eur. J. Med. Genet. 53, 227-233.

Knight, S., et al., 1999. Subtle chromosomal rearrangements in children with unexplained mental retardation. Lancet 354, 1676-1681.

Ruhi, H., et al., 2001. A Down syndrome case with a karyotype of 46, XY, rec(21) dup(21q)inv(21)(p11q22) derived from paternal pericentric inversion of chromosome 21. Clin. Genet. 59, 368-370.

Lazzaro, S.J., et al., 2001. Recombinant Down syndrome: a case report and literature review. Clin. Genet. 59, 128-130.

Gray, J.E., et al., 1962. Pericentric inversion of chromosome 21. A possible further cytogenetic mechanism in mongolism. Lancet 1, 21-23.

Fraisse, J., et al., 1986. Six cases of partial duplication-deficiency 21 syndrome: 21(dupq22-del23) due to maternal pericentric inversion: $\operatorname{inv}(21)(\mathrm{p} 12 ; \mathrm{q} 22)$. A family study. Ann. Genet. 29, 177-180.

Leonard, C., et al., 1986. Two Down's syndrome patients with rec(21), dupq, inv(21)(p11; q2109) from a familial pericentric inversion. Ann. Genet. 29, 181-183.

Miyazaki, K., et al., 1987. A boy with Down's syndrome having recombinant chromosome 21 but no SOD1 excess. Clin. Genet. 32, 383-387.

Tardy, E.P., et al., 1997. Prenatal exclusion of segmental trisomy in familial chromosome 21 pericentric inversion by fluorescence in situ hybridization. Prenat. Diagn. 17, 871-873.

Bennett, Robin L., et al., 1995. Recommendations for standardized human pedigree nomenclature. Am. J. Hum. Genet. 56, 745-752.

El-Badramany, M.H., et al., 1989. Familial manic-depressive illness with deleted short arm of chromosome 21: coincidental or causal? Br. J. Psychiatry 155, 856-857.

Chettouh, Z., et al., 1995. Molecular mapping of 21 features associated with partial monosomy 21: involvement of the APP-SOD I region. Am. J. Hum. Genet. 57, 62-71.

Roberson, E., et al., 2011. Genomic analysis of partial 21q monosomies with variable phenotypes. Eur. J. Hum. Genet. 19, 235-238

Valero, R., et al., 1999. Molecular characterisation of partial chromosome 21 aneuploidies by fluorescent PCR. J. Med. Genet. 36, 694-699.

Migdalska, A.M., et al., 2012. Modeling partial monosomy for human chromosome 21q11.2-q21.1 reveals haploinsufficient genes influencing behavior and fat deposition. PLoS One 7 (1), e29681. 